\title{
Comments on "An Organizational Theory of Information" by A.W. Holt and F. Cardone
}

\author{
Ronald K. Stamper \\ MEASURE Ltd, 29 Dartmouth Road Park, London NW5 $1 S U$ \\ Tel: +4402078137359 Fax: +4402074822968 \\ Email: rstamper@compuserve.com \\ University of Twente, P.O. Box 217, NL-7500 AE Enschede \\ Email: r.k.stamper@sms.utwente.nl
}

This paper is rather tough reading. Like FRISCO Chapter 4, it is dense, abstract and somewhat formal. The reader is advised to populate its abstractions with concrete instances. It would also be interesting to visit the corpus of raw material that inspired this approach to information systems. The authors have an abundance of varied practical experience to draw upon. One can often appreciate a theory better when its roots are visible, at least this guides one's choice of concrete illustrations.

Its style forces the reader to take a personal route into its meaning. For me, that route lies naturally enough through my semiotic framework. Every information system that functions successfully must be functioning on each of these levels. (See Section 6.1 of the FRISCO Report.) Thus, one way of interpreting this paper is to check how it handles each level.

Cardone and Holt begin with the physical level. The theory is grounded upon the notion of Organised Activity, OA, which developed among our biological ancestors, preceding language. 
Thus they provide us with an escape from the circularity of verbal definitions. Information and its phenomena can be brought into empirical relationships with aspects of $\mathrm{OA}$.

In the original version of their paper, the version I was invited to discuss, they go rather too far, in my opinion, in relating information to physical phenomena, when they claim information as a commodity. But information is fundamentally unlike goods that may be scarce commodities. For a trivial cost in material resources, any information can be reproduced. Information is only like a good and in limited supply when we conjure it to be so by means of the laws creating copyrights, patents, etc. This observation breaks the syllogism leading them from information being a commodity plus "all goods call crimes into existence" to the problem of the misuse of information. The same conclusion is reached directly if one adopts Eco's definition of a sign (and therefore information) as anything with which one can tell a lie. Information is the instrument for making and manipulating the social domain (=OA?), hence its value as a criminal instrument.

The authors capture a key to the empirics level neatly. By their assertion that OA always entails co-ordination and repetition. Co-ordination can only occur when there is some communication channel linking the co-ordinated elements. The central problems of empirics concern the transmission of signals through channels. This notion of co-ordination takes us further than the familiar model of

source $>$ encoder $>$ channel $>$ decoder $>$ receiver

because it forces us into an awareness of the completion of the cycle of transmission, if only via an observer. Within that transmission cycle, the empirical study of the properties of signs requires one to count the frequencies of the tokens sent and received, and their random transformations (= lack of co-ordination). The existence of countable signtokens is only possible when they are recognisably repeated forms falling into a classification. Hence, I am content that their conceptual framework is sufficient for this level of analysis.

They capture the core ideas of the syntactic level in at least two ways. Neatest of them is illustrated by the White Queen metaphor. Alice is confronted with syntactic alternatives for integers. On the semantic level they are the same. On the syntactic level they are different, as Alice indignantly points out. The thread of a syntactic invariant links them together and that link is a suitable notion of validity, preserved by deductive rules. 
The basis of the semantic properties of information is rooted the authors' notions of a unit and the relations of realisations, cases, parts and so on. These enable the universal / particular, the generic / specific and the part / whole structures to be developed. The production and consumption processes and the changes of state are handled in the spirit of classical physics without addressing the problem of explaining how we know of the connections between past and present, except through the use of information. Here, I think too much is taken for granted.

Pragmatics concerns intentional commitments, which are treated by the authors in terms of authentication. They emphasise the importance of identifying the communication, the maker and the receiver. Their notion of a plan also relates to pragmatics but this is embedded in the notion of the conceptual units, especially those constituting our social reality. Information, in their analysis, has to be understood in its context, a key component of which is the social activity and its associated plan. This is an excellent way of making sure (transformed into my perspective) that we insist that any phenomenon that embodies information must function correctly on every level of the semiotic framework. Eco's definition of a sign similarly captures its essence at the pragmatic level.

The social level constituted from the organised activity basis to this theory is the central idea. Language games are recognised as instruments for creating the 'units' in which the world is conceived. However, I am not convinced that "every organized activity is described by a plan". This would rule out those organised patterns of activity that are performed without conscious planning but express the norms which a community has evolved for those activities, such as those regulating behaviour in a crowded shopping centre, for example. If the plans do not have to be conscious, then perhaps they equate with norms.

My few remarks cannot begin to capture these authors' many challenges to our views and theories about information. The absence of the notion of a 'sign' is notable and was a subject of long discussions during the conference. I suspect that, once I can reinterpret their theory of organised activity in terms of norms and signs, I shall discover many more congruencies than conflicts. I recommend other readers to bring to this serious work their own co-ordinate frameworks, not necessarily mine. 